Supporting Information for:

\title{
Projected change in atmospheric nitrogen deposition to the Baltic Sea towards 2020
}

C. Geels ${ }^{1}$, K. M. Hansen ${ }^{1}$, J. H. Christensen ${ }^{1}$, C. Ambelas Skjøth ${ }^{2}$, T. Ellerman ${ }^{1}$, G. B. Hedegaard $^{1,3}$, O. Hertel ${ }^{1,4}$, L. M. Frohn ${ }^{1}$, A. Gross ${ }^{1}$, A. B. Hansen ${ }^{1}$ and J. Brandt ${ }^{1}$

[1] \{Department of Environmental Science, Aarhus University, Roskilde, Denmark\}

[2] \{Faculty of Science, Lund University, Sweden\}

[3] \{Danish Climate Center, Danish Meteorological Institute, Copenhagen, Denmark\}

[4] \{Department for Environmental, Social and Spatial Change, Roskilde University, Denmark \}

Correspondence to: K. M. Hansen ( $\underline{\mathrm{kmh} @ \mathrm{dmu} . \mathrm{dk}})$ or C. Geels (ag@ @dmu.dk $)$

One figure

Five tables 


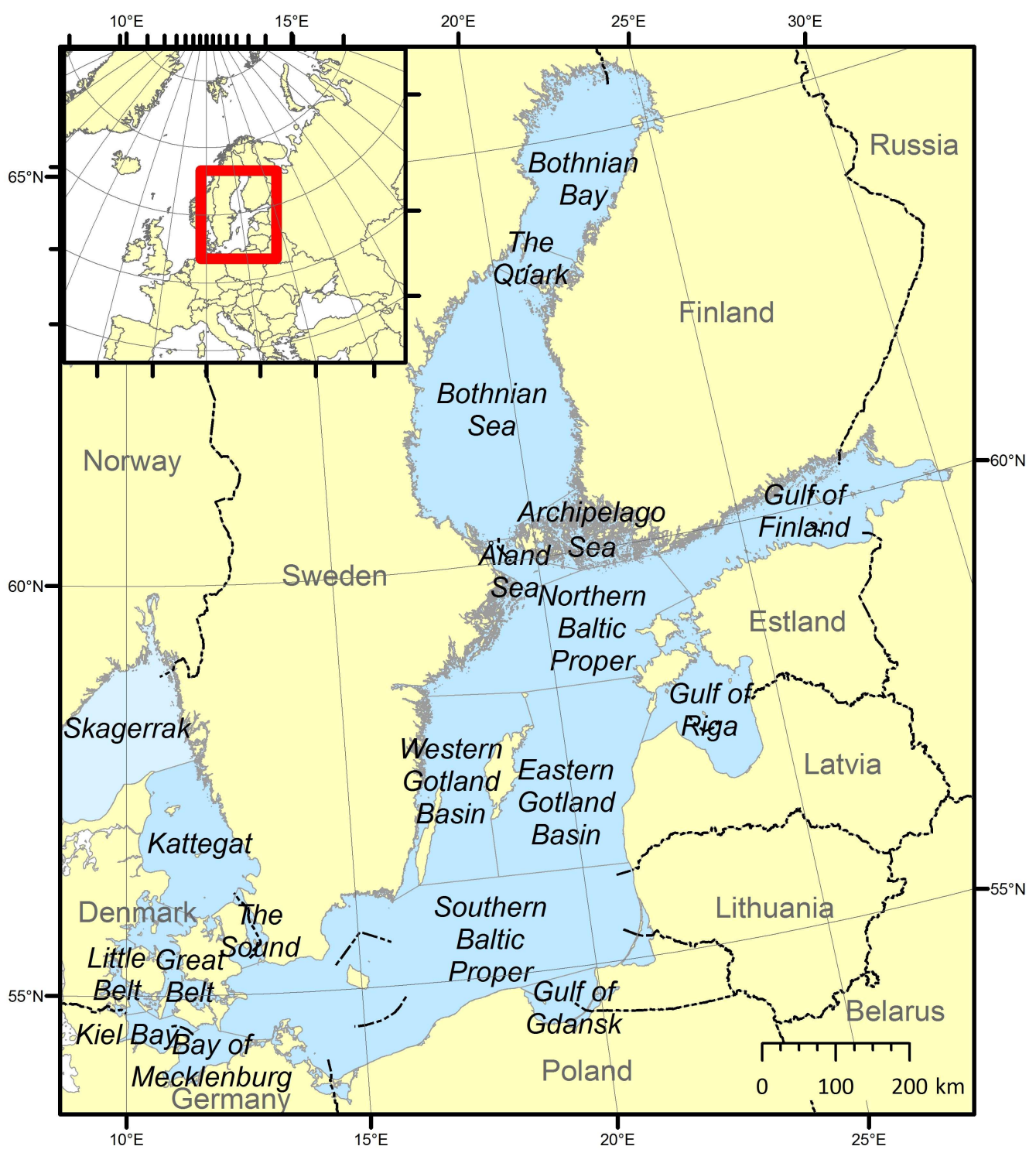

Figure S1. Map of the Baltic Sea with names of the different basins and sub-basins. 
Table S1. Calculated N depositions $\left[\mathrm{kg} / \mathrm{km}^{2}\right]$ to the basins and sub-basins of the Baltic Sea in 2007 for all countries in Europe and from the nine countries around the Baltic Sea as well as for ships. The Sea areas are defined in Figure S1.

\begin{tabular}{|c|c|c|c|c|c|c|c|c|c|c|c|c|c|c|c|c|c|c|c|c|c|c|c|c|}
\hline \multicolumn{2}{|l|}{ Area } & $\begin{array}{l}\text { All } \\
\text { Sources }\end{array}$ & \multicolumn{2}{|c|}{$\begin{array}{l}\Sigma \text { Baltic } \\
\text { countries }\end{array}$} & \multicolumn{2}{|c|}{ Denmark } & \multicolumn{2}{|c|}{ Germany } & \multicolumn{2}{|c|}{ Sweden } & \multicolumn{2}{|c|}{ Finland } & \multicolumn{2}{|c|}{ Poland } & Estonia & \multicolumn{2}{|r|}{ Latvia } & \multicolumn{2}{|c|}{ Lithuania } & \multicolumn{3}{|c|}{ Russia } & \multicolumn{2}{|c|}{ Ships } \\
\hline Gulf of $B$ & thnia & 304 & 148 & & 9 & & 22 & & 24 & & 33 & & 19 & & 4 & 3 & & 4 & & 30 & & & & \\
\hline & Bothnian Bay & 257 & & 15 & & 5 & & 13 & & 14 & & 39 & & 9 & & 2 & & 2 & & & & 29 & & 17 \\
\hline & The Quark & 265 & & 29 & & 5 & & 14 & & 17 & & 51 & & 1 & & 3 & & 2 & & & & 4 & & 20 \\
\hline & Bothnian Sea & 299 & & 51 & & 9 & & 22 & & 27 & & 29 & & 22 & & 4 & & 4 & & & & 30 & & 29 \\
\hline & Archipelago Sea & 457 & & 216 & & 18 & & 47 & & 28 & & 37 & & 32 & & 6 & & 5 & & & & 36 & & 45 \\
\hline & Åland Sea & 373 & & 189 & & 14 & & 32 & & 37 & & 20 & & 35 & & 5 & & 5 & & & & 35 & & 36 \\
\hline Gulf of Fir & land & 516 & 215 & & 9 & & 36 & & 13 & & 22 & & 30 & & 18 & 6 & & 6 & & 75 & & & 1 & \\
\hline Gulf of Ris & & 391 & 197 & & 13 & & 48 & & 15 & & 8 & & 39 & & 12 & & 25 & & 16 & 21 & & & 2 & \\
\hline Baltic Pro & & 533 & 283 & & 30 & & 98 & & 32 & & 8 & & 67 & & 3 & 5 & & & 11 & 25 & & & 7 & \\
\hline & Northern Baltic Proper & 411 & & 204 & & 15 & & 48 & & 30 & & 16 & & 39 & & 7 & & 6 & & & & 36 & & 41 \\
\hline & Eastern Gotland Basin & 469 & & 244 & & 22 & & 71 & & 33 & & 9 & & 50 & & 4 & & & 1 & & & 32 & & 50 \\
\hline & Western Gotland Basin & 450 & & 243 & & 20 & & 65 & & 52 & & 10 & & 16 & & 3 & & 5 & 1 & & & 33 & & 41 \\
\hline & Southern Baltic Proper & 631 & & 342 & & 44 & & 144 & & 27 & & 4 & & 35 & & 2 & & 3 & 1 & & & 23 & & 49 \\
\hline & Gulf of Gdansk & 705 & & 112 & & 24 & & 110 & & 16 & & 6 & & & & 2 & & 4 & 1 & & & 25 & & 45 \\
\hline The Soun & & 717 & 404 & & 145 & & 155 & & 44 & & 2 & & 40 & & 0 & 1 & & 3 & & 1 & & & & \\
\hline Belt Sea & & 988 & 606 & & 219 & & 325 & & 7 & & 1 & & 38 & & 1 & 1 & & 3 & & 1 & 2 & 4 & 7 & \\
\hline & Great Belt & 882 & & 540 & & 89 & & 184 & & 10 & & 1 & & 38 & & 1 & & 1 & & & & 13 & & 45 \\
\hline & Little Belt & 1218 & & 306 & & 98 & & 352 & & 6 & & 1 & & 35 & & 0 & & 1 & & & & 11 & & 49 \\
\hline & Bay of Mecklenburg & 948 & & 547 & & 66 & & 420 & & 5 & & 1 & & 0 & & 0 & & 1 & & & & 11 & & 47 \\
\hline & Kiel Bay & 1084 & & 66 & & 15 & & 493 & & 4 & & 1 & & 66 & & 1 & & 1 & & & & 2 & & 47 \\
\hline Kattegat & & 625 & 347 & & 165 & & 104 & & 30 & & 2 & & 30 & & 1 & 1 & & 3 & & & 2 & & 6 & \\
\hline
\end{tabular}

Table S2. Calculated N depositions $\left[\mathrm{kg} / \mathrm{km}^{2}\right]$ to the basins and sub-basins of the Baltic Sea in 2020 for all countries in Europe and from the nine countries around the Baltic Sea as well as for ships. The Sea areas are defined in Figure S1.

\begin{tabular}{|c|c|c|c|c|c|c|c|c|c|c|c|c|c|}
\hline Area & $\begin{array}{l}\text { All } \\
\text { Sources }\end{array}$ & $\begin{array}{l}\text { B Baltic } \\
\text { countries }\end{array}$ & Denmark & Germany & Sweden & Finland & Poland & Estonia & Latvia & Lithuania & Russia & Ships & \\
\hline Gulf of Bothnia & 258 & 111 & 5 & 13 & 15 & 23 & 12 & 2 & 2 & 3 & 35 & 27 & \\
\hline Bothnian Bay & 224 & 91 & 3 & 8 & 9 & 27 & 6 & 1 & 1 & 1 & 34 & & 17 \\
\hline The Quark & 227 & 100 & 3 & 9 & 11 & 38 & 7 & 2 & 1 & 2 & 28 & & 20 \\
\hline Bothnian Sea & 252 & 113 & 6 & 13 & 17 & 20 & 14 & 2 & 2 & 3 & 35 & & 29 \\
\hline Archipelago Sea & 382 & 158 & 11 & 29 & 18 & 27 & 20 & 4 & 3 & 5 & 41 & & 46 \\
\hline Åland Sea & 315 & 139 & 9 & 19 & 24 & 14 & 22 & 3 & 3 & 5 & 41 & & 37 \\
\hline Gulf of Finland & 464 & 170 & 6 & 22 & 8 & 15 & 20 & 12 & 4 & 5 & 79 & 33 & \\
\hline Gulf of Riga & 327 & 145 & 8 & 29 & 10 & 5 & 26 & 10 & 17 & 13 & 27 & 34 & \\
\hline Baltic Proper & 429 & 210 & 20 & 63 & 21 & 6 & 48 & 2 & 3 & 8 & 38 & 50 & \\
\hline Northern Baltic Proper & 342 & 148 & 9 & 30 & 19 & 11 & 24 & 4 & 4 & 6 & 41 & & 43 \\
\hline Eastern Gotland Basin & 382 & 177 & 13 & 43 & 21 & 6 & 33 & 3 & 6 & 11 & 40 & & 53 \\
\hline Western Gotland Basin & 368 & 176 & 12 & 40 & 34 & 7 & 30 & 2 & 3 & 7 & 40 & & 45 \\
\hline Southern Baltic Proper & 499 & 255 & 31 & 94 & 18 & 3 & 64 & 1 & 2 & 8 & 34 & & 53 \\
\hline Gulf of Gdansk & 591 & 334 & 15 & 68 & 10 & 4 & 177 & 1 & 3 & 8 & 48 & & 47 \\
\hline The Sound & 531 & 292 & 111 & 102 & 30 & 1 & 28 & 0 & 1 & 2 & 17 & 54 & \\
\hline Belt Sea & 734 & 444 & 164 & 228 & 5 & 1 & 28 & 0 & 1 & 2 & 15 & 53 & \\
\hline Great Belt & 656 & 396 & 217 & 124 & 7 & 1 & 28 & 0 & 1 & 2 & 16 & & 51 \\
\hline Little Belt & 898 & 588 & 294 & 247 & 4 & 1 & 26 & 0 & 1 & 2 & 14 & & 55 \\
\hline Bay of Mecklenburg & 706 & 400 & 49 & 299 & 3 & 1 & 31 & 0 & 1 & 2 & 14 & & 53 \\
\hline Kiel Bay & 807 & 489 & 86 & 355 & 3 & 1 & 27 & 0 & 1 & 2 & 15 & & 53 \\
\hline Kattegat & 460 & 247 & 121 & 66 & 19 & 2 & 20 & 0 & 1 & 2 & 16 & 62 & \\
\hline
\end{tabular}


Table S3. Calculated N depositions [t] to the basins and sub-basins of the Baltic Sea in 2007 for all countries in Europe and from the nine countries around the Baltic Sea as well as for ships. The Sea areas are defined in Figure S1.

\begin{tabular}{|c|c|c|c|c|c|c|c|c|c|c|c|c|}
\hline Area & \begin{tabular}{|l|l} 
All \\
Sources
\end{tabular} & $\begin{array}{l}\Sigma \text { Baltic } \\
\text { countries }\end{array}$ & Denmark & Germany & Sweden & Finland & Poland & Estonia & Latvia & Lithuania & Russia & Ships \\
\hline Gulf of Bothnia & 35503 & 17286 & 1044 & 2553 & 2768 & 3863 & 2238 & 431 & 367 & 484 & 3539 & 3144 \\
\hline Bothnian Bay & 8535 & 3832 & 158 & 443 & 481 & 1291 & 305 & 80 & 53 & 67 & 954 & 570 \\
\hline The Quark & 1197 & 584 & 22 & 64 & 77 & 230 & 49 & 14 & 10 & 11 & 108 & 89 \\
\hline Bothnian Sea & 19049 & 9636 & 597 & 1386 & 1738 & 1844 & 1374 & 251 & 229 & 299 & 1920 & 1830 \\
\hline Archipelago Sea & 5067 & 2396 & 204 & 520 & 310 & 409 & 355 & 65 & 55 & 75 & 404 & 496 \\
\hline Åland Sea & 1655 & 838 & 63 & 141 & 163 & 90 & 155 & 20 & 22 & 31 & 154 & 159 \\
\hline Gulf of Finland & 15447 & 6419 & 280 & 1063 & 387 & 651 & 886 & 527 & 173 & 194 & 2257 & 927 \\
\hline Gulf of Riga & 7365 & 3710 & 240 & 907 & 282 & 156 & 730 & 229 & 462 & 302 & 401 & 604 \\
\hline Baltic Proper & 110972 & 59023 & 6195 & 20523 & 6610 & 1765 & 13992 & 676 & 1110 & 2199 & 5954 & 9716 \\
\hline Northern Baltic Proper & 16921 & 8402 & 602 & 1981 & 1240 & 665 & 1608 & 269 & 248 & 327 & 1461 & 1690 \\
\hline Eastern Gotland Basin & 19916 & 10350 & 913 & 3000 & 1385 & 395 & 2135 & 173 & 403 & 605 & 1340 & 2115 \\
\hline Western Gotland Basin & 12607 & 6806 & 548 & 1813 & 1444 & 280 & 1298 & 86 & 153 & 273 & 911 & 1148 \\
\hline Southern Baltic Proper & 57428 & 31071 & 3994 & 13092 & 2450 & 392 & 7691 & 137 & 283 & 935 & 2097 & 4500 \\
\hline Gulf of Gdansk & 4098 & 2394 & 138 & 637 & 91 & 33 & 1259 & 10 & 23 & 58 & 145 & 262 \\
\hline The Sound & 1635 & 921 & 330 & 354 & 101 & 4 & 92 & 1 & 2 & 7 & 30 & 112 \\
\hline Belt Sea & 18503 & 11360 & 4111 & 6081 & 131 & 23 & 709 & 9 & 18 & 49 & 228 & 872 \\
\hline Great Belt & 6851 & 4197 & 2245 & 1428 & 78 & 11 & 298 & 4 & 9 & 24 & 100 & 350 \\
\hline Little Belt & 3588 & 2373 & 1171 & 1036 & 17 & 3 & 102 & 1 & 3 & 7 & 33 & 145 \\
\hline Bay of Mecklenburg & 4397 & 2537 & 305 & 1949 & 22 & 5 & 188 & 2 & 4 & 10 & 52 & 216 \\
\hline Kiel Bay & 3666 & 2253 & 390 & 1669 & 14 & 3 & 122 & 2 & 3 & 8 & 42 & 160 \\
\hline Kattegat & 13815 & 7661 & 3644 & 2290 & 656 & 48 & 652 & 11 & 26 & 63 & 270 & 1244 \\
\hline Baltic Sea & 203240 & 106381 & 15843 & 33772 & 10935 & 6510 & 19300 & 1885 & 2160 & 3298 & 12679 & 16619 \\
\hline Contribution in percent & & 52 & 8 & 17 & 5 & 3 & 9 & 1 & 1 & 2 & 6 & 8 \\
\hline
\end{tabular}

Table S4. Calculated N depositions [ $\mathrm{t}$ ] to the basins and sub-basins of the Baltic Sea in 2020 for all countries in Europe and from the nine countries around the Baltic Sea as well as for ships. The Sea areas are defined in Figure S1.

\begin{tabular}{|c|c|c|c|c|c|c|c|c|c|c|c|c|}
\hline Area & \begin{tabular}{|l} 
All \\
Sources
\end{tabular} & \begin{tabular}{|l|}
$\Sigma$ Baltic \\
countries \\
\end{tabular} & Denmark & Germany & Sweden & Finland & Poland & Estonia & Latvia & Lithuania & Russia & Ships \\
\hline Gulf of Bothnia & 30141 & 13002 & 625 & 1539 & 1735 & 2727 & 1416 & 258 & 227 & 334 & 4140 & 3168 \\
\hline Bothnian Bay & 7454 & 3014 & 93 & 265 & 294 & 906 & 197 & 44 & 33 & 47 & 1135 & 556 \\
\hline The Quark & 1025 & 451 & 13 & 39 & 48 & 170 & 31 & 8 & 6 & 8 & 128 & 88 \\
\hline Bothnian Sea & 16031 & 7168 & 357 & 829 & 1092 & 1286 & 871 & 151 & 141 & 206 & 2236 & 1852 \\
\hline Archipelago Sea & 4237 & 1753 & 124 & 320 & 197 & 302 & 221 & 43 & 34 & 52 & 460 & 507 \\
\hline Åland Sea & 1395 & 616 & 39 & 86 & 104 & 63 & 96 & 12 & 13 & 21 & 182 & 165 \\
\hline Gulf of Finland & 13894 & 5076 & 168 & 644 & 246 & 438 & 588 & 361 & 111 & 143 & 2377 & 981 \\
\hline Gulf of Riga & 6162 & 2729 & 147 & 549 & 180 & 102 & 491 & 183 & 326 & 243 & 508 & 642 \\
\hline Baltic Proper & 89439 & 43660 & 4150 & 13105 & 4359 & 1160 & 10066 & 445 & 718 & 1730 & 7927 & 10370 \\
\hline Northern Baltic Proper & 14065 & 6080 & 365 & 1215 & 797 & 445 & 1004 & 175 & 156 & 236 & 1688 & 1766 \\
\hline Eastern Gotland Basin & 16226 & 7529 & 568 & 1830 & 910 & 257 & 1390 & 113 & 271 & 482 & 1707 & 2241 \\
\hline Western Gotland Basin & 10309 & 4940 & 346 & 1123 & 960 & 183 & 851 & 56 & 97 & 202 & 1123 & 1252 \\
\hline Southern Baltic Proper & 45405 & 23172 & 2784 & 8544 & 1633 & 254 & 5792 & 93 & 179 & 765 & 3127 & 4838 \\
\hline Gulf of Gdansk & 3433 & 1939 & 87 & 394 & 59 & 21 & 1029 & 7 & 15 & 46 & 281 & 273 \\
\hline The Sound & 1212 & 666 & 253 & 232 & 68 & 3 & 65 & 1 & 2 & 5 & 38 & 123 \\
\hline Belt Sea & 13753 & 8318 & 3073 & 4276 & 85 & 16 & 534 & 7 & 11 & 38 & 277 & 989 \\
\hline Great Belt & 5100 & 3078 & 1690 & 962 & 51 & 8 & 217 & 3 & 5 & 18 & 122 & 399 \\
\hline Little Belt & 2645 & 1733 & 867 & 727 & 11 & 2 & 78 & 1 & 2 & 6 & 40 & 161 \\
\hline Bay of Mecklenburg & 3278 & 1855 & 227 & 1387 & 14 & 4 & 146 & 2 & 3 & 8 & 65 & 248 \\
\hline Kiel Bay & 2731 & 1653 & 290 & 1200 & 9 & 2 & 93 & 1 & 2 & 6 & 50 & 181 \\
\hline Kattegat & 10179 & 5468 & 2680 & 1454 & 430 & 34 & 445 & 9 & 17 & 50 & 349 & 1362 \\
\hline Baltic Sea & 164780 & 78920 & 11098 & 21799 & 7102 & 4480 & 13604 & 1264 & 1412 & 2544 & 15616 & 17634 \\
\hline Contribution in percent & & 48 & 7 & 13 & 4 & 3 & 8 & 1 & 1 & 2 & 9 & 11 \\
\hline
\end{tabular}


Table S5. Calculated reductions in N depositions (in \% of 2007 depositions) to the basins and subbasins of the Baltic Sea in 2020 compared to 2007 for all countries in Europe and from the nine countries around the Baltic Sea. The Sea areas are defined in Figure S1.

\begin{tabular}{|c|c|c|c|c|c|c|c|c|c|c|c|c|}
\hline Area & \begin{tabular}{|l} 
All \\
Sources
\end{tabular} & $\begin{array}{l}\Sigma \text { Baltic } \\
\text { countries }\end{array}$ & Denmark & Germany & Sweden & Finland & Poland & Estonia & Latvia & Lithuania & Russia & Ships \\
\hline Gulf of Bothnia & 15 & 25 & 40 & 40 & 37 & 29 & 37 & 40 & 38 & 31 & -17 & -1 \\
\hline Bothnian Bay & 13 & 21 & 41 & 40 & 39 & 30 & 35 & 45 & 38 & 30 & -19 & 3 \\
\hline The Quark & 14 & 23 & 41 & 39 & 38 & 26 & 37 & 39 & 37 & 30 & -18 & c \\
\hline Bothnian Sea & 16 & 26 & 40 & 40 & 37 & 30 & 37 & 40 & 38 & 31 & -16 & -1 \\
\hline Archipelago Sea & 16 & 27 & 39 & 38 & 36 & 26 & 38 & 35 & 38 & 31 & -14 & -2 \\
\hline Åland Sea & 16 & 26 & 39 & 39 & 36 & 30 & 38 & 39 & 38 & 32 & -18 & -4 \\
\hline Gulf of Finland & 10 & 21 & 40 & 39 & 36 & 33 & 34 & 31 & 36 & 26 & -5 & -6 \\
\hline Gulf of Riga & 16 & 26 & 39 & 39 & 36 & 35 & 33 & 20 & 29 & 20 & -27 & -6 \\
\hline Baltic Proper & 19 & 26 & 33 & 36 & 34 & 34 & 28 & 34 & 35 & 21 & -33 & -7 \\
\hline Northern Baltic Proper & 17 & 28 & 39 & 39 & 36 & 33 & 38 & 35 & 37 & 28 & -16 & -4 \\
\hline Eastern Gotland Basin & 19 & 27 & 38 & 39 & 34 & 35 & 35 & 35 & 33 & 20 & -27 & -6 \\
\hline Western Gotland Basin & 18 & 27 & 37 & 38 & 34 & 35 & 34 & 35 & 36 & 26 & -23 & $-\varsigma$ \\
\hline Southern Baltic Proper & 21 & 25 & 30 & 35 & 33 & 35 & 25 & 32 & 37 & 18 & -49 & $-\varepsilon$ \\
\hline Gulf of Gdansk & 16 & 19 & 37 & 38 & 36 & 36 & 18 & 29 & 37 & 20 & -95 & -4 \\
\hline The Sound & 26 & 28 & 23 & 34 & 33 & 29 & 29 & 19 & 37 & 23 & -27 & -10 \\
\hline Belt Sea & 26 & 27 & 25 & 30 & 35 & 29 & 25 & 25 & 38 & 23 & -22 & -13 \\
\hline Great Belt & 26 & 27 & 25 & 33 & 34 & 28 & 27 & 22 & 37 & 23 & -22 & -14 \\
\hline Little Belt & 26 & 27 & 26 & 30 & 36 & 27 & 24 & 27 & 37 & 22 & -22 & -11 \\
\hline Bay of Mecklenburg & 25 & 27 & 26 & 29 & 35 & 30 & 22 & 24 & 38 & 23 & -23 & -14 \\
\hline Kiel Bay & 26 & 27 & 26 & 28 & 36 & 29 & 24 & 31 & 38 & 23 & -19 & -13 \\
\hline Kattegat & 26 & 29 & 26 & 37 & 34 & 28 & 32 & 22 & 36 & 21 & -29 & -9 \\
\hline Baltic Sea & 19 & 26 & 30 & 35 & 35 & 31 & 30 & 33 & 35 & 23 & -23 & -6 \\
\hline
\end{tabular}

